\title{
Motivación y burnout en profesores de educación física: incidencia de la frustración de las necesidades psicológicas básicas
}

\author{
Motivation and burnout in physical education teachers: \\ incidence of thwarting of the basic psychological needs
}

\author{
Motivação e burnout nos profesores de educação física: \\ incidência da frustração das necessidades psicológicas básicas
}

\author{
David Sánchez-Oliva ${ }^{1}$, Pedro Antonio Sánchez-Miguel ${ }^{1}$, Juan José Pulido González ${ }^{1}$, José María López Chamorro ${ }^{1}$ y Ricardo \\ Cuevas Campos ${ }^{2}$ \\ ${ }^{1}$ Universidad de Extremadura (España), ${ }^{2}$ Universidad de Castilla-La Mancha (España)
}

\begin{abstract}
Resumen: A través del presente trabajo se pretende valorar la importancia de las necesidades psicológicas básicas para explicar y predecir el tipo de motivación y los niveles de burnout en profesores de educación física. Para ello, la muestra estuvo formada por 357 docentes de género masculino y femenino, pertenecientes a diferentes centros educativos públicos y privados de Espańa. Se realizó un análisis de regresión lineal por cada variable dependiente, que indicaron cómo la frustración de la necesidad de competencia predecía positivamente la desmotivación, el agotamiento emocional y el cinismo, y negativamente la motivación intrínseca y la eficacia profesional; la frustración de autonomía predecía positivamente el agotamientos emocional y negativamente la eficacia profesional; y la frustración de relaciones sociales predecía positivamente la eficacia profesional. Estos resultados están en la línea de la Teoría de la Autodeterminación (Deci y Ryan, 2000; Ryan y Deci, 2000), destacando la importancia que adquieren la satisfacción de autonomía, competencia y relaciones sociales para optimizar la motivación y el bienestar de los profesores de educación física.

Palabras clave: docentes, procesos motivacionales, agotamiento emocional, adolescencia.

Abstract: This work aims to assess the importance of the basic psychological needs to explain and predict the type of motivation and levels of burnout in physical education teachers. To achieve this purpose, the sample was formed by 357 male and female teachers, belonged to different public and private schools from Spain. A regression analysis by each dependent variable was conducted, which showed that thwarting of the needs of competence positively predicted amotivation, emotional burnout and effrontery, and negatively predicted intrinsic motivation and professional efficacy;
\end{abstract}

thwarting of the need of autonomy positively predicted emotional burnout and negatively predicted professional efficacy. These results are consistent with Self-Determination Theory (Deci y Ryan, 2000; Ryan y Deci, 2000), emphasizing the importance of satisfaction of autonomy, competence and relatedness to optimize motivation and welfare in physical education teachers.

Keywords: teachers, motivational processes, emotional exhaustion, adolescence.

Resumo: Através do presente trabalho pretendia-se avaliar a importância das necessidades psicológicas básicas para explicar e predizer o tipo de motivação e os níveis de burnout em professores de educação física. Para isso, a amostra foi formada por 357 professores do género masculino e feminino, pertencentes a diferentes centros educativos públicos e privadas de Espanha. Realizou-se uma análise de regressão linear para cada variável dependente, que indicaram como a frustraçáo da necessidade de competência previa positivamente a desmotivação, exaustão emocional e o cinismo e negativamente a motivação intrínseca e a eficácia profissional; a frustração de autonomia previa positivamente a exaustão emocional e negativamente a eficácia profissional; e a frustração das relaçôes sociais, previa positivamente, a eficácia profissional. Estes resultados estão na linha da Teoria da Autodeterminação (Deci y Ryan, 2000; Ryan y Deci, 2000), salientando a importância adquirida pela satisfaçáo de autonomia, competência e relaçóes sociais para optimizar a motivação e o bem-estar dos professores de educação física.

Palavras chave: professores, processos motivacionais, exaustão emocional, a adolescência.

\section{Introducción}

La adecuada motivación y el bienestar de los profesores son factores psicológicos necesarios para un correcto rendimiento profesional. En este sentido, bajos niveles de motivación y malestar docente se han relacionado con consecuencias ne-

Dirección para correspondencia [Correspodence address]: David Sánchez Oliva. Facultad de Ciencias del Deporte. Universidad de Extremadura. Avenida de la Universidad, s/n. 10071. Cáceres (España). E-mail: davidsanchez@unex.es gativas como descenso en la calidad de la enseñanza (Maslach y Leiter, 2005), absentismo, falta de preparación de las clases, bajos niveles de empatía hacia los alumnos, o escaso compromiso con la profesión docente (Burke, Greenglass, y Schwarzer, 1996).

El análisis de la salud psicológica de los profesores resulta especialmente necesario en el caso de los docentes de educación física $(\mathrm{EF})$, ya que por la idiosincrasia de su asignatura 
se encuentran expuestos a fuentes de estrés adicionales a las de materias de carácter más conceptual. Así, el constante movimiento del alumnado en clase, el bajo estatus social entre el colectivo docente o la falta de instalaciones adecuadas (Sáenz-López, Almagro, y Ibáńez, 2011), pueden derivar en un descenso de la motivación y la aparición de agotamiento físico y mental entre estos profesionales. Siguiendo el modelo jerárquico de la motivación de Vallerand (2007), los factores contextuales que, en este caso están referidos al centro de trabajo de los docentes, influyen directamente en el tipo de motivación y en la salud psicológica de los individuos. Por ende, el presente trabajo pretende conocer cómo la frustración de las necesidades psicológicas básicas (NPB) influye directamente en el tipo de motivación y en la posibilidad de experimentar burnout en profesores de EF.

El síndrome de burnout puede definirse como estrés laboral crónico excesivo que provoca una disminución de la habilidad de desempeńo de la actividad profesional y la aparición de diferentes problemas de salud en quien lo padece (Maslach, Schaufeli, y Leiter, 2001). Este estado de malestar psicológico derivado del estrés, se caracteriza por tres factores principales: cansancio emocional, despersonalización o cinismo, y falta de realización profesional (Schaufeli, Leiter, Maslach, y Jackson, 1996). El cansancio emocional se caracteriza por fatiga mental y falta de recursos emocionales para afrontar situaciones laborales. La despersonalización se refiere a la falta de empatía y el cinismo en las interacciones sociales en el trabajo. Y por último, la baja realización profesional se concreta en falta de eficacia en la ejecución de las tareas del trabajo.

Durante los últimos años, son varios los estudios que han constatado los altos niveles de burnout en docentes de EF. Por ejemplo, un estudio realizado recientemente en Portugal reveló que el $60,6 \%$ de los profesores presentaban un alto agotamiento emocional, el 22,3\% una alta despersonalización y el 34,0\% una baja realización profesional (Sinott, Afonso, Ribeiro, y Farias, 2014). Asimismo, otros trabajos también revelaron niveles relativamente altos de burnout en este colectivo profesional en España (Bartholomew, Ntoumanis, Cuevas, y Lonsdale, 2014), Italia (Carraro, Scarpa, Gobbi, Bertollo, y Robazza, 2010), Bélgica (Van den Berghe et al., en prensa), Venezuela (Viloria, Paredes, y Paredes, 2003) o Noruega (Skaalvik y Skaalvik, 2011).

Por otro lado, la presencia o ausencia de burnout en un individuo está relacionada con el tipo de motivación que posee el mismo. Para analizar el tipo de regulación motivacional, la teoría de la Autodeterminación (Deci y Ryan, 2000; Ryan y Deci, 2000) ha sido ampliamente utilizada en el contexto deportivo (Standage y Ryan, 2012), educativo (Ryan y Deci, 2009) o laboral (Vansteenkiste, Lens, Witte, Witte, y Deci, 2004), tratando de explicar los motivos por los cuales las personas se involucran en una determinada actividad. Esta teoría explicita en qué medida los comportamientos son volitivos o autodeterminados, estableciendo un continuo motivacional en función del nivel de autodeterminación, estableciendo tres grandes bloques motivacionales que son la motivación intrínseca, la motivación extrínseca y la desmotivación. La motivación intrínseca supone el nivel más alto de autodeterminación, y está relacionada con personas que se involucran en una actividad por motivos intrínsecos, como pueden ser la satisfacción, el disfrute o el placer. La motivación extrínseca supone el segundo tipo de motivación, y se asocia a personas involucradas en una actividad por razones instrumentales, como puede ser la búsqueda de contingencias externas (a nivel económico, social...), por lo que los motivos de práctica no son inherentes a la propia actividad. A su vez, la motivación extrínseca se subdivide en cuatro tipos de regulación, que de mayor a menor autodeterminación son las regulaciones integrada, identificada, introyectada y externa. Por último, el nivel más bajo de autodeterminación se denomina desmotivación, en la que el sujeto no encuentra motivos (ni intrínsecos ni extrínsecos) para involucrarse en el desarrollo de la actividad.

De manera paralela a la explicación de los diferentes tipos de regulación motivacional, la teoría de la Autodeterminación también explica la existencia de una serie de "nutrientes psicológicos" que pueden promover la aparición de una motivación autodeterminada. Estos nutrientes son conocidos como necesidades psicológicas básicas, y son la necesidad de autonomía, competencia y relaciones sociales (Ryan y Deci, 2002). Concretamente, la necesidad de autonomía se refiere al deseo de las personas de ser el origen de sus comportamientos, y por lo tanto, está relacionada con la libertad otorgada para tomar decisiones mientras se realiza la actividad. Del mismo modo, la necesidad de competencia hace referencia a la sensación de las personas de actuar eficazmente en el ambiente que le rodea con el objetivo de desarrollar sentimientos de logro. Por último, la necesidad de relaciones sociales responde a la pretensión de las personas de sentirse afiliados y pertenecientes a los grupos sociales que les rodean. Sin embargo, tal y como explican ampliamente Deci y Ryan (2000) en los postulados de su teoría, en la medida que las personas tengan una adecuada satisfacción de sus necesidades psicológicas básicas, se producirá un aumento del nivel de motivación autodeterminada.

Pero estas necesidades también pueden ser minadas por un ambiente negativo. Por ello, recientemente se ha desarrollado el constructo psicológico de frustración de las necesidades psicológicas (Bartholomew, Ntoumanis, Ryan, y ThøgersenNtoumani, 2011), referido a la sensación negativa experimentada por un individuo cuando aprecia que sus necesidades psicológicas están siendo activamente limitadas por la acción de otros significativos. La frustración de dichas necesidades es un concepto disímil a la satisfacción de las mismas (Bartholomew et al., 2011). Por ejemplo, un profesor puede no 
tener buenas relaciones sociales con sus compañeros porque no se siente cercano y ni tiene afinidades comunes con ellos; sin embargo, otro docente puede no tener buenas relaciones con sus colegas porque éstos le rechazan. Obviamente, ambos casos son diferentes: en el primer caso nos referimos de baja satisfacción de la necesidad de relación, mientras que en el segundo estaríamos hablando de la frustración de dicha necesidad. Por ende, para valorar la frustración de las necesidades psicológicas resulta necesario hacer referencia al efecto que provocan los otros significativos en dichas necesidades.

En cuanto a los estudios que han realizado hasta el momento, lo cierto es que tradicionalmente, la gran mayoría de los trabajos desarrollados en el contexto de la EF se limitaban a la inclusión de variables referentes a los estudiantes. Sin embargo, en los últimos años ha aparecido una nueva corriente que trata de analizar también los procesos motivacionales desarrollados por los profesores de EF, y cómo estas variables pueden determinar la aparición de consecuencias referentes a los estudiantes (estilo de enseñanza y estilo de liderazgo), o referentes a los propios profesores (burnout, satisfacción con la vida...).

Por ejemplo, Taylor, Ntoumanis, y Standage (2008) realizaron un estudio con 204 profesores de EF, en el que comprobaron cómo las tres NPB correlacionaban positiva y significativamente con la motivación autodeterminada. Además, estos autores desarrollaron un modelo de ecuaciones estructurales, en el que la satisfacción global de las NPB predijo significativamente la motivación autodeterminada. Posteriormente, Carson y Chase (2009) analizaron la motivación de 247 profesores de EF, comprobando cómo los docentes que tenían una mayor satisfacción de sus necesidades de autonomía, competencia y relaciones sociales se correspondían con los aquellos profesores que tenían mayores niveles en la motivación intrínseca y por el contrario menores niveles de desmotivación. De igual modo, Bartholomew, Ntoumanis, Cuevas, y Lonsdale (2014) realizaron un estudio con 364 profesores de EF, a través del cual pudieron comprobar cómo aquellos profesores que tenían una mayor frustración de sus necesidades de autonomía, competencia y relaciones sociales eran los que desarrollaban mayores niveles de burnout. Paralelamente, Van den Berghe et al. (en prensa) llevaron a cabo un estudio con 201 profesores de EF, en el que encontraron que las tres NPB se relacionaban positivamente con las regulaciones intrínseca e identificada, mientras que las satisfacciones de autonomía y competencia se asociaron negativamente con la regulación externa. Además, la satisfacción de las necesidades de autonomía, competencia y relaciones se relacionó positivamente con la eficacia profesional y negativamente con el agotamiento emocional y la despersonalización.

A pesar del creciente interés por conocer los procesos motivacionales desarrollados por los profesores de EF, resulta necesario seguir investigando en aras de mejorar el bienestar y la calidad de la docencia de los profesores (Bartholomew et al., 2014). De esta manera, este trabajo permite profundizar en el análisis de la importancia que adquieren las NPB en el ambiente laboral, pues analiza la capacidad predictiva de la frustración de las tres necesidades sobre la motivación intrínseca, la desmotivación, así como sobre las tres dimensiones que conforman el burnout. Por tanto, a través de este estudio se pretendió analizar la incidencia de la frustración de las NPB sobre los sentimientos de burnout y el tipo de motivación en los profesores de EF. Como hipótesis de trabajo, se planteó que aquellos profesores que tuvieran frustradas sus necesidades de autonomía, competencia y relación tendrían mayores niveles de agotamiento emocional, cinismo y desmotivación, mientras que desarrollarían una mayor eficacia profesional y motivación intrínseca.

\section{Método}

\section{Participantes}

Participaron en el estudio 357 profesores de EF, de género masculino $(\mathrm{n}=231)$ y femenino $(\mathrm{n}=126)$, con una experiencia profesional entre 1 y 41 ańos $(M=15,31$; DT = 9,23). Todos los profesores fueron seleccionados a través de muestreo simple aleatorio, e impartían clases en centros públicos $(\mathrm{n}=$ 280) y concertados $(n=77)$ en todo el territorio nacional.

\section{Instrumentos}

Frustración de las NPB. Para analizar la frustración de las necesidades de autonomía, competencia y relaciones sociales, se utilizó la adaptación al contexto educativo español (Cuevas, Ntoumanis, Sanchez-Oliva, Bartholomew, y Garcia-Calvo, 2012) de la Psychological Need Thwarting Scale (PNTS: Bartholomew, Ntoumanis, Ryan, y Thøgersen-Ntoumani, 2011). Este cuestionario está formado por la frase inicial "En mi ambiente de trabajo...", seguida de 12 ítems (4 por factor) encaminados a analizar la frustración de autonomía (ejemplo: Siento que me impiden tomar decisiones respecto al modo en el que enseño), la frustración de competencia (ejemplo: Hay situaciones que me hacen sentir incapaz) y la frustración de relaciones sociales (ejemplo: Siento que soy rechazado por aquellos que me rodean). Las propiedades psicométricas de esta escala en el contexto educativo han sido demostradas en trabajos previos (Bartholomew et al., 2014; Cuevas et al., 2012). En el presente trabajo, el cuestionario registro valores en el alfa de Cronbach de ,74 para la Frustración de Autonomía, ,78 para la Frustración de Competencia y ,69 para la Frustración de Relaciones Sociales.

Tipo de motivación. Para analizar los niveles de motivación intrínseca y desmotivación, se utilizaron los factores correspondientes del Work Motivation Inventory (WMI; 
Blais, Lachance, Vallerand, Briere, y Riddle, 1993). Esta escala estaba introducida por la pregunta "iPor qué me dedico a la enseńanza?", y cada uno de los factores estaba compuesto por 4 ítems: motivación intrínseca (ejemplo: "Por los grandes momentos de placer que me proporciona la enseñanza") y desmotivación (ejemplo: "No sé por qué, estamos provistos de condiciones de trabajo surrealistas"). En el presente trabajo, la Motivación Intrínseca obtuvo un alfa de Cronbach de ,81, mientras que la Desmotivación registró un valor de ,65.

Burnout. Con el objetivo de analizar los niveles burnout en los profesores, se empleó versión en castellano (Gil-Monte, 2002) del Maslach Burnout Inventory-General (MBI-GS; Schaufeli, Leiter, Maslach, y Jackson, 1996). Este cuestionario está precedido por la frase "En relación a mis sensaciones en el trabajo...", seguida de ítems encaminados a evaluar el Agotamiento Emocional (6 ítems; ejemplo: "Debido a mi trabajo me siento emocionalmente agotado"), el Cinismo (5 ítems; ejemplo: "Dudo de la importancia de mi trabajo") y la Eficacia Profesional (6 ítems; ejemplo: "Creo que he logrado muchas cosas que valen la pena en este trabajo"). En el estudio del burnout, la eficacia profesional se ha valorado en otras ocasiones de forma negativa a través de la puntuación inversa de los ítems. En este trabajo se ha procedido a valorar esta dimensión sin invertir la puntuación de la escala. Respecto a la consistencia interna obtenida en este estudio, se obtuvieron valores de .84 para Agotamiento Emocional, ,74 en el Cinismo y, 70 en la Eficacia Profesional.

Todos factores del estudio fueron tratados como variables continuas, y el formato de respuesta empleado en todos los instrumentos fue una escala tipo Likert en la que cada ítem tenía un rango de respuesta de 1 a 5 . El 1 se correspondía con totalmente en desacuerdo y el 5 con totalmente de acuerdo con la formulación de la pregunta.

\section{Procedimiento}

Para llevar a cabo la recogida de datos se desarrolló un protocolo de actuación para que la obtención de resultados fuera similar en todos los participantes. Para ello, la recogida de los datos se realizó vía telemática, a través del software Google Docs ${ }^{1}$. Concretamente, se envió un correo electrónico a los profesores en el que se explicaban los objetivos de la investigación, así como la aceptación para forma parte del estudio. Además, en dicho correo electrónico se facilitaba un enlace web a través del cual los profesores participantes podían acceder telemáticamente para completar el cuestionario.

\footnotetext{
1 Google Docs es un software libre que permite crear cuestionarios on-line, pudiendo acceder al documento a través de un enlace URL. Una vez completado el cuestionario, el software almacena los datos en un documento Excel.
}

\section{Análisis de los datos}

En primer lugar, se calcularon los estadísticos descriptivos (Media y Desviación Típica) de las diferentes variables incluidas en el estudio, así como la consistencia interna de los instrumentos a través del coeficiente Alfa de Cronbach. Posteriormente, se realizó un análisis de correlaciones bivariadas a través del Coeficientes de Correlación de Pearson. Por último, se realizó un análisis de regresión lineal utilizando el método introducir, estimando el cambio producido en $\mathrm{R}^{2}$, así como las estimaciones de cada variable predictora sobre la variable dependiente. Todos los análisis fueron realizados con el programa SPSS en versión 19,0.

\section{Resultados}

\section{Análisis Descriptivo}

En primer lugar, se analizaron los estadísticos descriptivos de las diferentes variables incluidas en el estudio. Como se puede apreciar en la Tabla 1, los profesores mostraron medias bajas en la frustración de sus NPB, siendo las relaciones sociales la variable que obtuvo la puntuación más baja y la autonomía la puntuación más elevada. Del mismo modo, los profesores obtuvieron puntuaciones altas en la motivación intrínseca y bajas en la desmotivación. Por último, respecto a los factores del burnout, la eficacia profesional registró la media más elevada del estudio, mientras que el agotamiento emocional y el cinismo obtuvieron medias bajas.

Tabla 1. Estadísticos descriptivos.

\begin{tabular}{llll}
\cline { 2 - 4 } & $M$ & $D T$ & Rango \\
\hline Frustración Autonomía & 1,51 &, 65 & $1-5$ \\
Frustración Competencia & 1,55 &, 71 & $1-5$ \\
Frustración Relaciones Sociales & 1,46 &, 60 & $1-5$ \\
Motivación Intrínseca & 3,82 &, 63 & $1-5$ \\
Desmotivación & 1,94 &, 57 & $1-5$ \\
Agotamiento Emocional & 2,13 &, 82 & $1-5$ \\
Cinismo & 1,84 &, 73 & $1-5$ \\
Eficacia Profesional & 4,25 &, 54 & $1-5$ \\
\hline
\end{tabular}

\section{Análisis Correlacional}

En segundo lugar, se realizó un análisis de correlaciones bivariadas incluyendo todas las variables incluidas en el estudio. La motivación intrínseca se relacionó negativamente con la frustración de competencia, mientras que la desmotivación se relacionó positivamente con la frustración de autonomía, competencia y relaciones sociales. Por último, 
el agotamiento emocional y el cinismo se asociaron de forma negativa con la frustración de autonomía, competencia y relaciones sociales, ocurriendo a la inversa en el caso de la eficacia profesional.

Tabla 2. Análisis Correlacional.

\begin{tabular}{lccc}
\cline { 2 - 4 } & $\begin{array}{c}\text { Frustración } \\
\text { Autonomía }\end{array}$ & $\begin{array}{c}\text { Frustración } \\
\text { Competencia }\end{array}$ & $\begin{array}{c}\text { Frustración } \\
\text { Relaciones } \\
\text { Sociales }\end{array}$ \\
\hline Motivación Intrínseca &,- 08 &,$- 14^{* *}$ &,- 07 \\
$\begin{array}{l}\text { Desmotivación } \\
\begin{array}{l}\text { Agotamiento Emo- } \\
\text { cional }\end{array}\end{array}$ &, $38^{* *}$ &, $41^{* *}$ &, $37^{* *}$ \\
$\begin{array}{l}\text { Cinismo } \\
\text { Eficacia Profesional }\end{array}$ &, $33^{* *}$ &, $46^{* *}$ &, $38^{* *}$ \\
\hline
\end{tabular}

${ }^{*}=p<, 05 ;{ }^{* *}=p<, 01$.

\section{Análisis de regresión}

Se realizó un análisis de regresión mediante el método introducir, incluyendo en este caso la motivación intrínseca y la desmotivación como variables dependientes. La varianzas explicadas fueron del $3 \%$ para la motivación intrínseca y del $18 \%$ para la desmotivación. La frustración de competencia predijo negativamente la motivación intrínseca, mientras que destacó como predictor positivo de la desmotivación.

Tabla 3. Análisis de regresión.

\begin{tabular}{|c|c|c|c|c|}
\hline \multirow[b]{3}{*}{ Variables predictoras } & \multicolumn{4}{|c|}{ Variables Dependientes } \\
\hline & \multicolumn{2}{|c|}{$\begin{array}{l}\text { Motivación } \\
\text { Intrínseca }\end{array}$} & \multicolumn{2}{|c|}{ Desmotivación } \\
\hline & $\beta$ & $p$ & $\beta$ & $p$ \\
\hline & \multicolumn{2}{|c|}{$R^{2}=, 03$} & \multicolumn{2}{|c|}{$R^{2}=, 18$} \\
\hline Frustración Autonomía & ,07 & ,47 &, 11 &, 25 \\
\hline Frustración Competencia &,- 27 &, $01^{* *}$ &, 25 &, $01^{* *}$ \\
\hline Frustración Relaciones Sociales & ,09 & ,36 &, 10 & ,26 \\
\hline
\end{tabular}

Posteriormente, se realizó un nuevo análisis de regresión, incluyendo como variables dependientes los factores referentes al burnout y como variables predictoras la frustración de las tres NPB. Se obtuvo una varianza explicada del $22 \%$ para el agotamiento emocional, 21\% para el cinismo y $15 \%$ para la eficacia profesional. Como se aprecia en la Tabla 3, las frustraciones de autonomía y competencia destacaron como predictores positivos del agotamiento emocional, mientras que la frustración de competencia predijo positivamente el cinismo. Por el contrario, las frustraciones de autonomía y competencia predijeron negativamente la eficacia profesional, ocurriendo a la inversa en el caso de la frustración de relaciones sociales.

Tabla 4. Análisis de regresión.

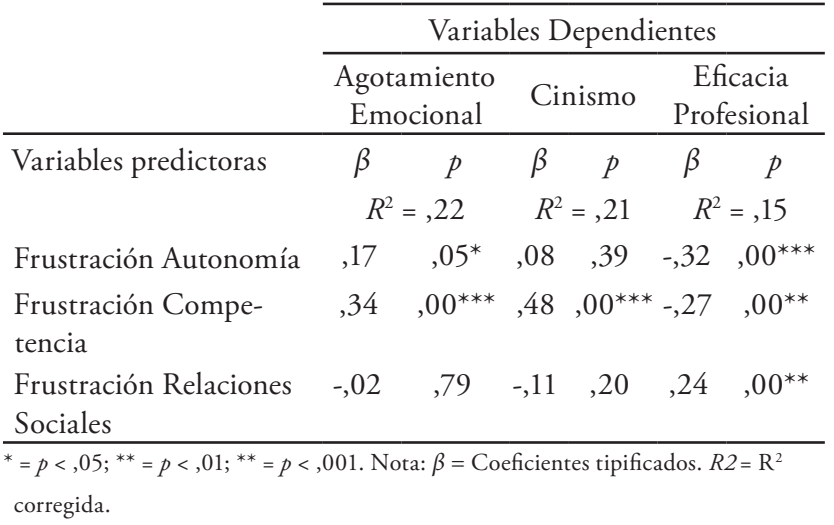

\section{Discusión}

El objetivo de este estudio era comprobar la incidencia de la frustración de las NPB sobre el tipo de motivación y el burnout en profesores de EF. Los resultados encontrados ratifican los postulados de la teoría de la Autodeterminación (Deci y Ryan, 2000; Ryan y Deci, 2000), comprobándose la importancia que adquieren la satisfacción de autonomía, competencia y relaciones sociales para optimizar la motivación y la disposición hacia el trabajo.

En primer lugar, respecto a los resultados descriptivos obtenidos, cabe destacar cómo los profesores pertenecientes al estudio registraron valores óptimos en cuanto a los procesos motivacionales y el burnout. Concretamente, obtuvieron valores bajos en la frustración de las NPB, la desmotivación y el cinismo, y valores altos en la motivación intrínseca y la eficacia profesional. Tan sólo el agotamiento emocional registró un valor superior a lo ideal, aspecto que debe ser tenido en cuenta, ya que es un resultado consistente con trabajos anteriores en los que también registraron valores considerables en el agotamiento emocional (Carraro et al., 2010; Van Den Berghe et al., 2013; Van den Berghe et al., en prensa; Viloria et al., 2003)

Por otro lado, el principal objetivo de este trabajo era conocer la incidencia que la frustración de las NPB podía tener sobre el tipo de motivación y los niveles de burnout. En primer lugar, los resultados destacaron la importancia que la percepción de competencia tiene sobre el tipo de motivación hacia el trabajo. Concretamente aquellos profesores que tienen una mayor frustración de su competencia son los que estaban más desmotivados hacia el trabajo, y además 
mostraban menores niveles en la motivación intrínseca. Es decir, el hecho de que los docentes se sientan que no se les deja demostrar su competencia (sentirse incapaz por la acción de sus jefes, dificultades docentes...) puede provocar la aparición de sentimientos de desmotivación hacia su labor docente, pudiendo llegar incluso a preguntar el por qué dedicarse a su labor docente. Como consecuencia parece lógico pensar que aquellos profesores con una baja percepción de competencia hacia el trabajo sean los que desarrollen menores niveles de motivación intrínseca, como pueden ser el disfrute, la satisfacción o el placer.

No existen trabajos previos que permitan comparar la incidencia de la frustración de las NPB sobre el tipo de motivación. Así, tan sólo se han encontrado estudios que incluyen la satisfacción de las necesidades, como los trabajos desarrollados por Taylor et al. (2008) y Van den Berghe et al. (2013), en los que comprobaron cómo los profesores que tenían una mayor satisfacción de sus NPB eran los que presentaban una motivación más autodeterminada hacia el trabajo, o el estudio llevado a cabo por Carson y Chase (2009), en el que encontraron que las satisfacciones de autonomía, competencia y relaciones sociales se asociaban positivamente a la motivación intrínseca (siendo las relaciones sociales el predictor más fuerte), mientras que no encontraron relaciones destacables con la desmotivación.

Por otro lado, el análisis de regresión también evidenció la importancia que adquieren las NPB para explicar y predecir los niveles de burnout. Concretamente, la frustración de autonomía predijo positivamente el agotamiento emocional y negativamente la eficacia profesional, es decir, aquellos profesores que sienten que les impiden tomar ciertas decisiones en su labor como docentes son los que desarrollan mayores niveles de estrés psicológico y además, son los que tiene una menor eficacia en el trabajo. Con esto, se destaca la necesidad de ceder autonomía a los docentes en cuento a los aspectos relacionados con la docencia, como pueden ser la organización de la asignatura, la evaluación o el estilo de enseñanza y liderazgo a utilizar. Además, la frustración de competencia fue el principal predictor del burnout, asociándose positivamente con el agotamiento emocional y el cinismo y negativamente con la eficacia profesional. Con este hallazgo, nuevamente se subraya la importancia que adquiere crear ambientes de trabajo en los que los docentes puedan demostrar su potencial profesional, evitando con ello la aparición de situaciones de estrés en el trabajo. Por último, la frustración de relaciones sociales emergió como predictor positivo de la eficacia profesional, es decir, aquellos profesores que no tienen una buena relación con sus compañeros de trabajo son los que muestran una mayor eficacia en el trabajo. A pesar de que el nivel de predicción con respecto a las otras necesidades psicológicas es menor, este es un resultado no esperado. En este caso, los datos nos podrían indicar que las relaciones del profesorado con sus compañeros no son tan importantes para su bienestar como se conjeturó. Así, tendría un mayor impacto en su salud psicológica la sensación de competencia y autonomía en el trabajo que las buenas relaciones sociales. No obstante, esta afirmación deberá ser contrastada por futuros estudios.

En cuanto a los trabajos previos, los resultados coinciden con el estudio de Bartholomew et al. (2014) en el que también comprobaron cómo la frustración de autonomía, competencia y relaciones sociales predecían positivamente el burnout, aunque en este caso la frustración de autonomía destacó como mayor predictor. También en la misma línea, Van Den Berghe et al. (2013) comprobaron cómo la satisfacción de las tres NPB se asoció positivamente a los factores negativos del burnout y negativamente a la eficacia profesional.

En definitiva, este trabajo ha permitido profundizar en el análisis de la importancia que adquieren las NPB en el ambiente escolar, para explicar y predecir el tipo de motivación y los niveles de Burnout. En este sentido, la totalidad de estudios realizados hasta el momento, o bien se limitaban a la realización de análisis de correlaciones bivariadas, o bien realizaban modelos de ecuaciones estructurales uniendo los tres factores del burnout. Así, el presente trabajo permite ahondar en el conocimiento de los procesos motivacionales desarrollados por los profesores de EF, analizando de manera separada la incidencia de las NPB sobre cada una de las variables dependientes incluidas.

No obstante, a pesar de los hallazgos, este trabajo presenta una serie de limitaciones. En primer lugar, el modo de selección de los participantes hace aconsejar nuevos estudios que confirmen los resultados. Asimismo, sería recomendable la implementación de investigaciones longitudinales con profesores para analizar, como ya se ha realizado con deportistas (Balaguer et al., 2012), las posibles relaciones de causa y efecto entre la frustración de las necesidades psicológicas y el burnout.

\section{Aplicaciones prácticas}

El presente trabajo ha permitido destacar la importancia que adquieren las NPB en la labor diaria del profesor de EF. Así, se sugiere la creación de ambientes de trabajo basados en el apoyo a las necesidades psicológicas en los centros educativos. Especialmente se aconseja la implementación de condiciones adecuadas para que el profesorado de los centros pueda demostrar su potencial profesional, por la estrecha relación que tiene la frustración de la necesidad de competencia con el burnout. De este modo, los responsables de la administración educativa y los directores de los centros estarán favoreciendo la salud psicológica y el bienestar de sus docentes lo que, a su vez, facilitaría un mayor rendimiento profesional y una educación de mayor calidad para los discentes. 


\section{Referencias}

1. Balaguer, I., González, L., Fabra, P., Castillo, I., Mercé, J., y Duda, J. L. (2012). Coaches' interpersonal style, basic psychological needs and the well- and ill-being of young soccer players: A longitudinal analysis. Journal of Sports Sciences, 30, 1619-1629.

2. Bartholomew, K. J., Ntoumanis, N., Cuevas, R., y Lonsdale, C. (2014). Job pressure and ill-health in physical education teachers: The mediating role of psychological need thwarting. Teaching and Teacher Education, 37, 101-107.

3. Bartholomew, K. J., Ntoumanis, N., Ryan, R. M., y Thøgersen-Ntoumani, C. (2011). Psychological need thwarting in the sport context: assessing the darker side of athletic experience. Journal of Sport y Exercise Psychology, 33, 75-102.

4. Blais, M. R., Lachance, L., Vallerand, R. J., Briere, N. M., y Riddle, A. S. (1993). The work motivation inventory. Revue Quebecoise de Psychologie, 14, 185-215.

5. Burke, R. J., Greenglass, E. R., y Schwarzer, R. (1996). Predicting teacher burnout over time: Effects of work stress, social support, and selfdoubts on burnout and its consequences. Anxiety, Stress, and Coping, 9, 261-275.

6. Carraro, A., Scarpa, S., Gobbi, E., Bertollo, M., y Robazza, C. (2010). Burnout and Self-Perceptions of Physical Fitness in a Sample of Italian Physical Education Teachers. Perceptual and Motor Skills, 111, 790-798.

7. Carson, R. L., y Chase, M. A. (2009). An examination of physical education teacher motivation from a self-determination theoretical framework. Physical Education y Sport Pedagogy, 14, 335-353.

8. Cuevas, R., Ntoumanis, N., Sánchez-Oliva, D., Bartholomew, K. J., y Garcia-Calvo, T. (2012). Adaptation and validation of the psychological need thwarting scale to the Spanish context. En C. Arce y G. Seoane (Eds.), V European Congress of Methodology (pp. 180-181). Santiago de Compostela: University of Santiago de Compostela Publications.

9. Deci, E. L., y Ryan, R. M. (2000). The "What" and "Why" of goal pursuits: Human needs and the self-determination of behavior. Psychological Inquiry, 11, 227-268.

10. Gil-Monte, P. R. (2002). The factorial validity of the Maslach Burnout inventorygeneral survey (MBI-GS) Spanish version. Salud Publica de Mexico, 44, 33-40.

11. Maslach, C., y Leiter, M. P. (2005). Stress and burnout: The critical research. Handbook of Stress Medicine and Health, 2, 155-172.

12. Maslach, C., Schaufeli, W. B., y Leiter, M. P. (2001). Job Burnout. Annual Review of Psychology, 52, 397-422.

13. Ryan, R. M., y Deci, E. L. (2000). Self-determination theory and the facilitation of intrinsic motivation, social development, and well-being. The American Psychologist, 55, 68-78.

14. Ryan, R. M., y Deci, E. L. (2002). Overview of self-determination theory: An organismic dialectical perspective. En E. L. Deci y R. M.
Ryan (Eds.), Handbook of selfdetermination research (pp. 3-33). Rochester, NY: Rochester University Press.

15. Ryan, R. M., y Deci, E. L. (2009). Promoting self-determined school engagement: Motivation, learning, and well-being. En K. R. Wentzel y A. Wigfield (Eds.), Handbook on motivation at school (pp. 171-196). New York: Routledge.

16. Sáenz-lópez, P., Almagro, B. J., y Ibáñez, S. J. (2011). Describing Problems Experienced by Spanish Novice Physical Education Teachers. The Open Sports Sciences Journal, 4, 1-9.

17. Schaufeli, W. B., Leiter, M. P., Maslach, C., y Jackson, S. E. (1996). The MBI-general survey. En M. P. Leiter, C. Maslach, y S. E. Jackson (Eds.), Maslach Burnout inventory. (pp. 19-26). Palo Alto. CA: Consulting Psychologists Press.

18. Sinott, E. C., Afonso, M. R., Ribeiro, J. A. B., y Farias, G. O. (2014) Síndrome de Burnout: Um estudo com professores de Educação Física. Movimento, 20, 519-539.

19. Skaalvik, E. M., y Skaalvik, S. (2011). Teacher job satisfaction and motivation to leave the teaching profession: Relations with school context, feeling of belonging, and emotional exhaustion. Teaching and Teacher Education, 27, 1029-1038.

20. Standage, M., y Ryan, R. M. (2012). Self-determination theory and exercise motivation: Facilitating self-regulatory processes to support and maintain health and well-being. En G. C. Roberts y D. C. Treasure (Eds.), Advances in motivation in sport and exercise 3rd ed. (pp. 233-270). Champaign, IL: Human Kinetics.

21. Taylor, I. M., Ntoumanis, N., y Standage, M. (2008). A self-determination theory approach to understanding the antecedents of teachers' motivational strategies in physical education. Journal of Sport and Exercise Psychology, 30, 75-94.

22. Vallerand, R. J. (2007). Intrinsic and extrinsic motivation in sport and physical activity. En N. Singer, H. A. Hausenblas, y C. M. Janelle (Eds.), Handbook of sport Psychology (pp. 59-83). New York: Wiley.

23. Van Den Berghe, L., Cardon, G., Aelterman, N., Tallir, I. B., Vansteenkiste, M., y Haerens, L. (2013). Emotional Exhaustion and Motivation in Physical Education Teachers: A Variable- Centered and Person-Centered Approach. Journal of Teaching in Physical Education, 32, 305-320.

24. Van den Berghe, L., Soenens, B., Aelterman, N., Cardon, G., Tallir, I., y Haerens, L. (en prensa). Within-person profiles of teachers' motivation to teach: Associations with need satisfaction at work, need-supportive teaching, and burnout. Psychology of Sport and Exercise.

25. Vansteenkiste, M., Lens, W., Witte, S. D. E., Witte, H. D. E., y Deci, E. L. (2004). The "why" and "why not" of job search behaviour: Their relation to searching, unemployment experience, and well-being. European Journal of Social Psychology, 34, 345-363.

26. Viloria, H., Paredes, M., y Paredes, L. (2003). Burnout en profesores de educación física. Revista de Psicología Del Deporte, 12, 133-146. 